OPEN ACCESS

Edited by:

Caoxing Huang,

Nanjing Forestry University, China

Reviewed by:

Sibao Liu,

Tianjin University, China

Zhiwen Wang,

University of Groningen, Netherlands

${ }^{*}$ Correspondence:

Changzhi Li

licz@dicp.ac.cn

Li Shuai

lishuai@fafu.edu.cn

${ }^{\text {t}}$ These authors have contributed equally to this work

Specialty section: This article was submitted to

Bioprocess Engineering,

a section of the journal Frontiers in Bioengineering and

Biotechnology

Received: 10 January 2022 Accepted: 24 January 2022 Published: 15 February 2022

Citation:

Lv X, Luo X, Cheng X, Liu J, Li C and Shuai $L$ (2022) Production of Hydroxymethylfurfural Derivatives

From Furfural Derivatives via Hydroxymethylation.

Front. Bioeng. Biotechnol. 10:851668. doi: $10.3389 /$ fbioe.2022.851668

\section{Production of Hydroxymethylfurfural Derivatives From Furfural Derivatives via Hydroxymethylation}

\author{
Xianqing $\mathrm{Lv}^{1 \dagger}$, Xiaolin $\mathrm{LuO}^{1 \dagger}$, Xin Cheng ${ }^{1}$, Jing $\mathrm{Liu}^{1}$, Changzhi $\mathrm{Li}^{2 *}$ and $\mathrm{Li}$ Shuai ${ }^{1 *}$ \\ ${ }^{1}$ College of Materials Engineering, Fujian Agriculture and Forestry University, Fuzhou, China, ${ }^{2}$ CAS Key Laboratory of Science and \\ Technology on Applied Catalysis, Dalian Institute of Chemical Physics, Chinese Academy of Sciences, Dalian, China
}

Hydroxymethylfurfural (HMF) derivatives such as 2,5-bis(hydroxymethyl)furan (BHMF) and furandicarboxylic acid (FDCA) are promising alternative of fossil-based diols and dicarboxylic acids for synthesis of polyesters such as polyethylene terephthalate (PET). However, high cost for preparing HMF from biomass discourages the commercialization of HMF-derived polyesters. Since producing furfural (FUR) from five-carbon sugars (e.g., xylose) via dehydration is an inexpensive and commercialized process, we herein reported a method to synthesize BHMF derivatives (5-(ethoxymethyl)furan-2-methanol (EMFM), 2,5-bis(hydroxymethyl)furan monoacetate (BHMFM) and 2,5-bis(hydroxymethyl)furan diacetate (BHMFD) from furfural derivatives, i.e., (2-(ethoxymethyl)furan (EMF) and furfuryl acetate $(F A))$. To avoid strong acid-induced side reactions (e.g., furan ring opening, condensation and carbonization), two reaction systems, i.e., a lowconcentration $\mathrm{HCl}$ aqueous solution combined with formaldehyde and anhydrous acetic acid combined with paraformaldehyde, were found to be suitable for such a hydroxymethylation reaction and could lead to decent product yields. In order to improve the carbon utilization, condensed furanic byproducts were further converted into hydrocarbon fuels via a reported two-step hydrodeoxygenation (HDO) process. This study not only validates the possibility of synthesizing functional HMF derivatives (EMFM, BHMFM, and BHMFD) from commercially-available FUR derivatives (EMF and FA), but also provide a new way to transform condensed furanics to value-added hydrocarbon fuels.

Keywords: furfural derivatives, hydroxymethylfurfual derivatives, hydroxymethylation, hydrocarbon fuel, hydrodeoxygenation

\section{INTRODUCTION}

Synthetic polymers are important material basis for promoting the development of industry and human society. Most of the commercialized polymers [e.g., polyethylene terephthalate (PET)] are synthesized based on fossil-based chemicals (e.g., terephthalic acid). However, the extensive use of fossil-based chemicals brings about severe environmental problems such as the greenhouse effect and the shortage of nonrenewable resources. Developing alternatives of fossil-based chemicals and materials from renewable biomass is a promising way to overcome or at least alleviate these problems. For example, 2, 5-furandicarboxylic acid (FDCA) can be used to substitute terephthalic acid for synthesis of polyesters such as polyethylene furandicarboxylate (PEF), which showed better performance (e.g., thermostability and elasticity modulus) than that of PET (Guan et al., 2021; Yang and $\mathrm{Mu}, 2021$ ). Polyesters could also be synthesized by using 2,5-bis(hydroxymethyl)furan (BHMF) 


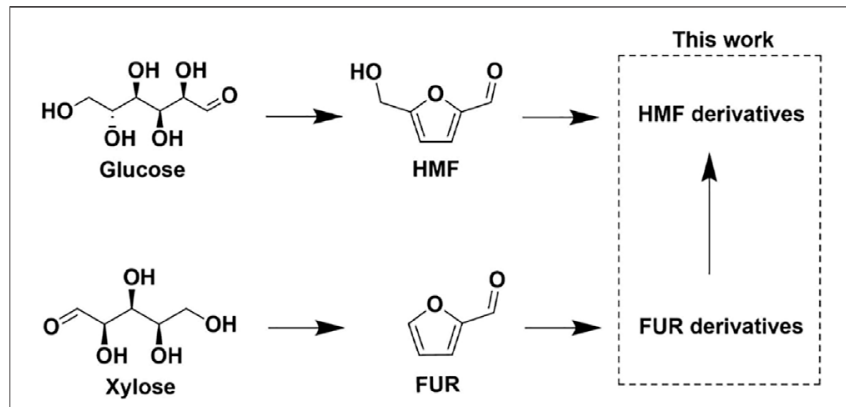

SCHEME 1 | The pathway of preparing HMF and HMF derivatives from biomass.

as an alternative of fossil-based diols (e.g., ethylene glycol). As a result, hydroxymethylfurfural (HMF) derivatives such as FDCA and BHMF have been considered as important platform chemicals for synthesizing bio-based polymers, which thereby stimulate intensive studies in preparing the two chemicals from renewable biomass (Scheme 1).

At present, FDCA is synthesized from 5-hydroxymethyl furfural (HMF) through hydrothermal (Gao et al., 2017; Cheng et al., 2021; Kandasamy et al., 2021; Yu et al., 2021) or electrochemical oxidation (Hu et al., 2021; Zhang et al., 2021). During the hydrothermal oxidation process, HMF is initially oxidized to 2, 5-diformylfuran (DFF) and/or 5-hydroxymethyl2-furanoic acid (HMFCA), which is further converted to 5formyl-2-furanoic acid (FFCA) and FDCA under aerobic conditions (Kandasamy et al., 2021). The reaction pathway of converting HMF to FDCA via electrochemical oxidation is similar to that of hydrothermal oxidation, but such a method showed good selectivity only at low substrate concentrations. In addition to FDCA, HMF can be also reductively converted to BHMF, which is a furanic diol for synthesis of polyesters. With the input of $\mathrm{H}_{2}$, different catalysts such as $\mathrm{Ru} / \mathrm{Co}_{3} \mathrm{O}_{4}$ (Chen et al., 2021), $\mathrm{Cu}-\mathrm{Al}_{2} \mathrm{O}_{3}$ (Rao et al., 2021), Ni-Cu/HT (Gupta et al., 2020), and $\mathrm{CuO}-\mathrm{Fe}_{3} \mathrm{O}_{4} / \mathrm{AC}$ (Elsayed et al., 2020), had been developed for reducing $\mathrm{HMF}$ to BHMF. These studies indicate that HMF and its derivatives are important bio-based small molecules for synthesizing FDCA, BHMF and many other value-added derivatives. However, a critical issue related to $\mathrm{HMF}$ and its derivatives is the high cost for preparing them from biomass. HMF is generally produced via the dehydration of six-carbon carbohydrates (e.g. glucose and fructose) while the dehydration process is either inefficient due to low selectivity of HMF or costly due to energy-intensive solvent recovery and separation (Sajid et al., 2018). In contrast, dehydration of fivecarbon sugars (e.g. xylose) to produce furfural (FUR) is a commercialized process and furfural has been successfully used to synthesize furan resin (Ye et al., 2021).

As the production of FUR from five-carbon sugars such as xylose is easy and inexpensive, we believe that synthesis of HMF or HMF derivatives (e.g. BHMF ethers and esters) from inexpensive FUR or its derivatives (e.g., furfuryl acetate and 2(ethoxymethyl)furan)) would be an interesting and potential way for further investigation. Such a pathway requires incorporation of one carbon to the C-5 position of furfural via hydroxymethylation, which was rarely reported. Therefore, in this article we proposed new reaction pathways for synthesizing BHMF derivatives (2,5-bis(hydroxymethyl)furan diacetate and 5(ethoxymethyl)furan-2-methanol) via hydroxymethylation of furfuryl alcohol derivatives (furfuryl acetate and 2(ethoxymethyl)furan) with formaldehyde or paraformaldehyde (Figures 1A, 2A). The reason we chose etherified or esterified furfural derivatives as starting materials was that these derivatives were more stable than FUR and furfuryl alcohol under acidic conditions. During these reactions, we found that acid could lead to the opening of furan rings, which produced carbonyl compounds that would further condense to form humins-like products. To improve the utilization efficiency of bio-based carbon resources, the oxygen-containing condensed products were converted to hydrocarbon fuels via hydrodeoxygenation. These results validate not only a new way to synthesize HMF derivatives but also a new method for utilizing condensed furan products for hydrocarbon fuels production.

\section{MATERIALS AND METHODS}

\section{Materials}

Furfuryl acetate (99\%) and 2-(ethoxymethyl)furan (98\%) were purchased from Rhawn Chemical Technology Co., Ltd. (Shanghai, China). Paraformaldehyde (98\%), $\mathrm{Na}_{2} \mathrm{HPO}_{4} \cdot \mathrm{H}_{2} \mathrm{O}$ (98\%) and $\mathrm{NaH}_{2} \mathrm{PO}_{4}$ (98\%) were purchased from Maclin Biochemical Co., Ltd. (Shanghai, China). Pt on carbon (Pt $5 \mathrm{wt}$ $\%)$, n-hexane (98\%), n-hexadecane (99.5\%, GC), chloroform-d (99.8\%), formic acid (99\%), trifluoromethanesulfonic acid (98\%) and $\mathrm{MgSO}_{4}$ (AR) were ordered from Aladdin ${ }^{\circledR}$ Biochemical Technology Co., Ltd. (Shanghai, China). Phosphotungstic acid anhydrate (99\%) was purchased from Titan Scientific Co., Ltd. (Shanghai, China). Anhydrous acetic acid (99.5\%), formaldehyde (37 wt\%), chloroform, concentrated sulfuric acid (98 wt\%) and concentrated hydrochloric acid (36 wt\%) were all obtained from XiLong Scientific Co., Ltd. (Shantou, China). All reagents were used as received without further purification.

\section{Synthesis of 5-(ethoxymethyl) furan-2-methanol}

Initially, $0.4 \mathrm{mmol}$ of 2-(ethoxymethyl)furan (EMF) was mixed with $10 \mathrm{mmol}$ of formaldehyde (37 wt\%), $1 \mathrm{ml}$ of 1,4-dioxane and $10 \mu \mathrm{L}$ of concentrated hydrochloric acid (36 wt $\%$ ) in a $15-\mathrm{ml}$ vial. The vial was placed in oil bath, magnetically stirred and heated to a desired temperature $\left(30-50{ }^{\circ} \mathrm{C}\right)$ for $1-8 \mathrm{~h}$. After the reaction, the vial was immediately taken out from the oil bath and cooled by tap water to room temperature. Around $0.25 \mathrm{mg}$ n-hexadecane was added into the reaction liquor as an internal standard and $50 \mu \mathrm{L}$ of the liquid was sampled and extracted by $1 \mathrm{ml}$ of chloroform. The extract was dehydrated by around $200 \mathrm{mg}$ anhydrous $\mathrm{MgSO}_{4}$. The resulting solution was analyzed by a gas chromatograph-mass spectrometer (GC-MS, SCION 436GCSQ, Techcomp group, Shanghai, China) to identify products and the identified products were further quantitatively analyzed by a 

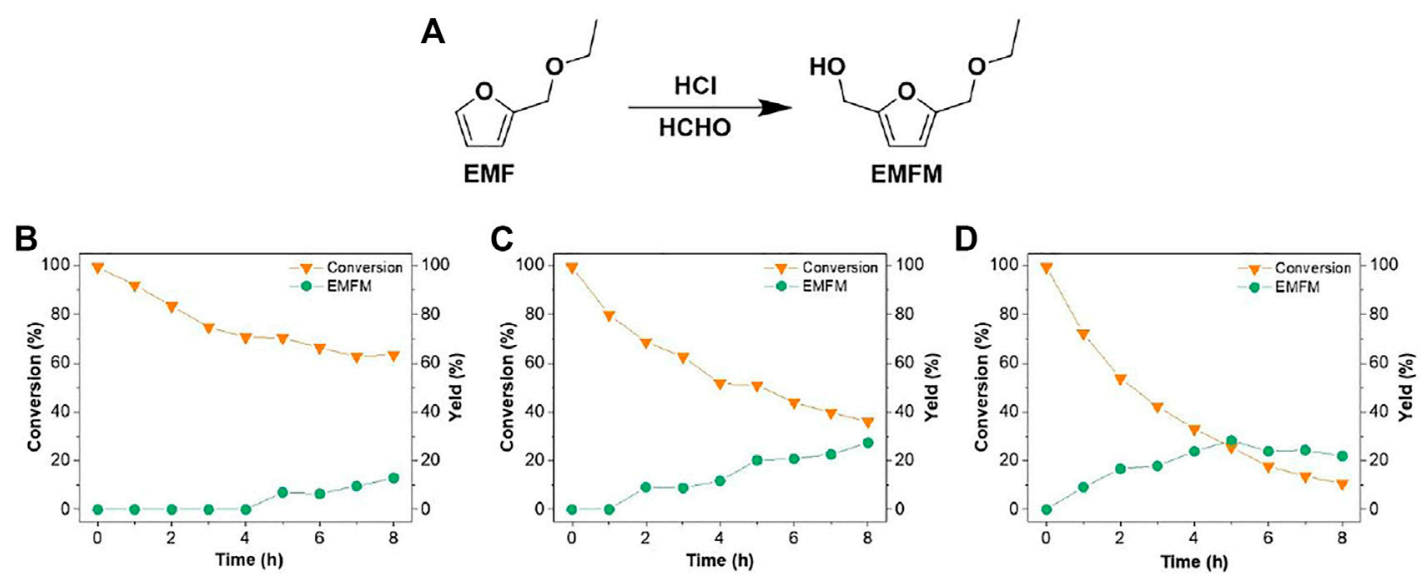

FIGURE 1 | (A) Synthesis pathway of EMFM from EMF; time-dependent conversion of EMF and yield of EMFM at (B) $30^{\circ} \mathrm{C}$; (C) $40^{\circ} \mathrm{C}$; and (D) $50^{\circ} \mathrm{C}$. Other hydroxylmethylation reaction conditions: $0.4 \mathrm{mmol} \mathrm{EMF}, 1 \mathrm{ml}$ formaldehyde ( $37 \mathrm{wt} \%$ aqueous solution), $0.1 \mathrm{mmol} \mathrm{HCl}(10 \mu \mathrm{L}$ of $36 \mathrm{wt} \% \mathrm{HCl}$ aqueous solution) and $1 \mathrm{ml} 1,4-$-dioxane.
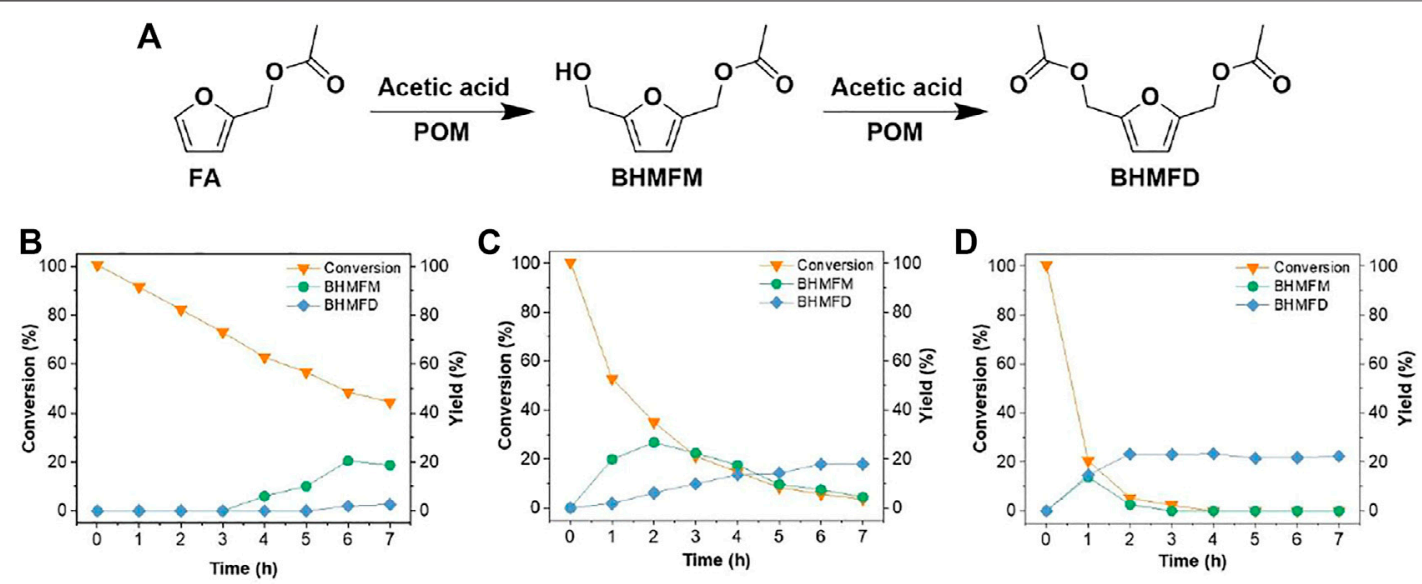

FIGURE 2 | (A) Synthesis pathway of BHMFM and BHMFD from FA; time-dependent conversion of FA and yield of BHMFM and BHMFD at (B) $80^{\circ} \mathrm{C}$; (C) $100^{\circ} \mathrm{C}$; and (D) $120^{\circ} \mathrm{C}$. Other hydroxylmethylation reaction conditions: $0.4 \mathrm{mmol} \mathrm{FA}, 1.7 \mathrm{mmol}$ paraformaldehyde and $35.0 \mathrm{mmol} \mathrm{CH}_{3} \mathrm{COOH}$.

gas chromatograph (GC, SCION 436C, Techcomp group, Shanghai, China).

\section{Synthesis of 2,5-bis(Hydroxymethyl)Furan Monoacetate (BHMFM) and 2,5-bis(Hydroxymethyl)Furan Diacetate (BHMFD)}

A mixture of furfuryl acetate (FA, $0.4 \mathrm{mmol})$, paraformaldehyde $(50 \mathrm{mg})$ and anhydrous acetic acid $(2 \mathrm{ml})$ was loaded into a 15$\mathrm{ml}$ vial. The vial was magnetically stirred and heated at $100-120^{\circ} \mathrm{C}$ in an oil bath for $1-7 \mathrm{~h}$. After the reaction, the vial was immediately taken out from the oil bath and cooled by tap water to room temperature. Around $0.25 \mathrm{mg}$ n-hexadecane was added into the reaction liquor as an internal standard. $50 \mu \mathrm{L}$ of the liquid was sampled and analyzed by a GC-MS (SCION 436GC-SQ, Techcomp group, Shanghai, China) to identify products and the identified products were further quantitatively analyzed by a GC (SCION 436C, Techcomp group, Shanghai, China).

\section{Separation of Monomeric Furanic Products From Condensed Furanics}

The solvent in the final product mixture was carefully removed with a rotary evaporator under vacuum. The monomeric furanic products in the residual mixture were extracted with $10 \mathrm{ml}$ hexane three times and the residual was considered as condensed furanics and used for oil production via hydrodeoxygenation.

\section{Hydrodeoxygenation of Condensed Furanics to Hydrocarbon Fuels}

The condensed furanics separated in last section was used as raw materials for production of hydrocarbon fuels (also termed as oil) through the following hydrodeoxygenation reactions. 
About $300 \mathrm{mg}$ of the residual product was re-dissolved with methanol $(25 \mathrm{ml})$ in a $50-\mathrm{ml}$ stainless steel reactor (WZD-50, Wuzhou Dingchuang Technology Co., Ltd., Beijing, China) followed by the addition of $\mathrm{Pt} / \mathrm{C}$ catalyst $(200 \mathrm{mg})$. The reactor was flushed three times with hydrogen gas, and then pressurized with hydrogen gas to $3 \mathrm{MPa}$. The mixture was mechanically stirred, and then heated to $250^{\circ} \mathrm{C}$ and kept at this temperature for $4 \mathrm{~h}$. After the reaction, the reactor was cooled to room temperature and depressurized carefully. The catalyst was separated by filtration, and the reaction liquor was collected. Methanol in the reaction liquor was removed by a vacuum rotary evaporator. The residual organics was further mixed with $\mathrm{Pt} / \mathrm{C}$ catalyst (200 mg), phosphotungstic acid (Li et al., 2021) (200 mg) and $\mathrm{n}$-hexane $(25 \mathrm{ml})$ in the reactor (WZD-50, Wuzhou Dingchuang Technology Co., Ltd., Beijing, China). The reactor was also flushed and pressurized with hydrogen gas to $3 \mathrm{MPa}$. The mixture was mechanically stirred at $300 \mathrm{rpm}$, and then heated to $250^{\circ} \mathrm{C}$ and reacted at the temperature for $4 \mathrm{~h}$. The reactor was cooled to room temperature and depressurized carefully after the reaction. After the catalyst was separated via filtration, n-hexane in the resultant filtrate was removed by a vacuum rotary evaporator. The resultant viscous oil was used for further characterization.

\section{Monomeric Furanics Purification and Charicterization \\ Purification of Monomeric Furanics}

Products obtained in the sections of "Synthesis of EMFM" and "Synthesis of BHMFM and BHMFD" were separated by a preparative liquid chromatograph (Sepabean machine2, Santai Technologies, Changzhou, China), which was equipped with a DAD detector and a Spherical C18 column (SW025, 20-45 $\mu \mathrm{m}$ ). The following separation conditions were used: $100 \%$ phosphate buffer $(\mathrm{pH} 6.5,10 \mathrm{mM}$ ) at $5 \mathrm{ml} / \mathrm{min}$ for $2 \mathrm{~min}$, increasing volumetric ratio of acetonitrile from 20 to $100 \%$ with $35 \mathrm{~min}$ and keeping flow rate of eluent at $5 \mathrm{ml} / \mathrm{min}, 100 \%$ acetonitrile at $5 \mathrm{ml} / \mathrm{min}$ for $10 \mathrm{~min}$ (McKenna et al., 2015). UV wavelengths at $225 \mathrm{~nm}$ for EMFM and at $230 \mathrm{~nm}$ for BHMFM and BHMFD collections were selected, respectively. Once a UV signal was detected, the fraction containing the targeted products was automatically collected. The structure of the collected fraction was identified by GC-MS, and then further analyzed by NMR.

\section{Identification of Monomeric Furanics}

Products separated by the preparative liquid chromatograph were initially identified by a GC-MS (SCION 436GC-SQ, Techcomp group, Shanghai, China) that was installed with a SCION-5 MS column $(30 \mathrm{~m} \times 0.32 \mathrm{~mm} \times 0.25 \mu \mathrm{m})$. The following temperature program was used for GC-MS analysis: the column temperature was initially set at $50^{\circ} \mathrm{C}$ and held at the temperature for $5 \mathrm{~min}$, heated at a rate of $10^{\circ} \mathrm{C} / \mathrm{min}$ to $300^{\circ} \mathrm{C}$ and held at $300^{\circ} \mathrm{C}$ for $5 \mathrm{~min}$.

After the solvent was removed from the fraction collected from the preparative liquid chromatograph, about $5-10 \mathrm{mg}$ of the purified product was dissolved in $0.5 \mathrm{ml}$ chloroform-d for nuclear magnetic resonance (NMR) spectrum analysis. All
${ }^{1} \mathrm{H}-\mathrm{NMR}$ spectra were acquired on a Bruker Ascend ${ }^{\mathrm{TM}} 600$ NMR Spectrum with an operating frequency of $600 \mathrm{MHz}$.

\section{Quantitation of Monomeric Furanics}

The products in the prepared samples (Sections of "Synthesis of EMFM" and "Synthesis of BHMFM and BHMFD") were quantitatively analyzed by GC (SCION 436C, Techcomp group, Shanghai, China). The following temperature program was used for GC analysis: the column temperature was initially set at $50^{\circ} \mathrm{C}$ and held at $50^{\circ} \mathrm{C}$ for $5 \mathrm{~min}$, then heated at a rate of $10^{\circ} \mathrm{C} /$ min to $300^{\circ} \mathrm{C}$ and held at $300^{\circ} \mathrm{C}$ for $5 \mathrm{~min}$.

The concentrations of reactants and products in chloroform solutions were quantified by a well-known effective carbon number method (Shuai et al., 2016). The conversion of reactants and the yield of products were thereby calculated as follows:

$$
\begin{gathered}
\text { Reactant conversion (\%) } \frac{\text { mol of reacted reactant }}{\text { Initial mol of reactant }} \times 100 \\
\text { Product yield (\%) } \frac{\text { mol of product }}{\text { Innitial mol of reactant }} \times 100
\end{gathered}
$$

\section{Elemental Analysis of Condensed Furanics Before and After Hydrodeoxygenation}

To evaluate the efficiency of hydrodeoxygenation reactions and the quality of hydrocarbon fuels, the contents (wt\%) of C and $\mathrm{H}$ elements in the prepared oil samples were analyzed by an element analyzer (Vario EL Cube, Elementar, Germany). Prior to the elemental analysis, solvent-free viscous oils obtained in the Section of "Hydrodeoxygenation of condensed furanics to hydrocarbon fuels" were further dried in a vacuum drying oven at $80^{\circ} \mathrm{C}$ for $48 \mathrm{~h}$ and then ground to powder in an agate mortar. The contents of $\mathrm{O}$ element in the samples was calculated by assuming that the total content of $\mathrm{C}, \mathrm{H}$ and $\mathrm{O}$ elements for each sample was $100 \%$.

\section{RESULTS AND DISCUSSION}

This study intends to introduce a hydroxymethyl group $\left(-\mathrm{CH}_{2} \mathrm{OH}\right)$ at the C-5 position of furan derivatives (2-(ethoxymethyl)furan (EMF) and furfuryl acetate (FA)) via electrophilic addition of formaldehyde for synthesizing 2,5-bis(hydroxymethyl)furan (BHMF) derivatives (5(ethoxymethyl)furan-2-methanol (EMFM), 2,5-bis(hydroxymethyl) furan monoacetate (BHMFM) and 2,5-bis(hydroxymethyl)furan diacetate (BHMFD)) (Figure 1A, Figure 2A). Because furanics are unstable under acidic conditions, the effects of different acids such as hydrochloric acid, sulfuric acid, formic acid, acetic acid and trifluoromethanesulfonic acid on the hydroxymethylations of EMF and FA were initially investigated.

\section{Synthesis of EMFM From EMF}

The results show that the strength of the acid in the reaction system has a great influence on the reaction. When the acidity used in the reaction medium was too high, the substrate was easily carbonized due to the acid-catalyzed ring opening of furan rings 
and severe condensation of the ring-opening products such as aldehydes or ketones. As such, strong acidity-induced carbonization reactions (e.g., entries $7,8,10$, and 11 in Supplementary Table S1; entries $3,4,6,8,9$, and 10 in Supplementary Table S2) resulted in dark colored reaction liquors and no hydroxymethylated product was detected in these reactions (Supplementary Table S1, Supplementary Table S2). Low-concentration hydrochloric or and anhydrous acetic acid exhibited better balance between acid-catalyzed hydroxymethylation and carbonization reactions, resulting in decent product yields. Therefore, these two catalytic systems were further studied for hydroxymethylation of furfuryl alcohol derivatives.

The results above demonstrated that low-concentration $\mathrm{HCl}$ aqueous solution was more beneficial than other reaction systems to facilitate the hydroxylmethylation reaction of EMF with formaldehyde. Since the acidity would be an important factor affecting the hydroxylmethylation reaction efficiency, the effect of $\mathrm{HCl}$ aqueous solution loading was thereby further investigated. 5(ethoxymethyl)furan-2-methanol

(EMFM),

a hydroxylmethylated product of EMF, was identified by MS (Supplementary Figure S1) and ${ }^{1} \mathrm{H}-\mathrm{NMR}$ (Supplementary Figure S2) and quantified by GC (Supplementary Figure S3), when the reaction between EMF $(0.4 \mathrm{mmol})$ and formaldehyde ( $1 \mathrm{ml}$ of $37 \mathrm{wt} \%$ aqueous solution, $10 \mathrm{mmol}$ ) was catalyzed by $10 \mu \mathrm{L}$ of $36 \mathrm{wt} \% \mathrm{HCl}$ aqueous solution $\left(0.1 \mathrm{mmol} \mathrm{H}^{+}\right)$in $1 \mathrm{ml}$ of 1,4-dioxane at $50^{\circ} \mathrm{C}$ for $7 \mathrm{~h}$. However, without the change of other reaction conditions, increasing the loading of $36 \mathrm{wt} \% \mathrm{HCl}$ aqueous solution to $20 \mu \mathrm{L}\left(0.2 \mathrm{mmol} \mathrm{H}^{+}\right)$resulted in a dark reaction solution and reduced hydroxylmethylated product. This could be mainly caused by the increased concentration of $\mathrm{H}^{+}$in water and 1, 4-dioxane mixture, which could accelerate the ring opening reactions of furanics (EMF and/or EMFM) and the condensation (e.g., aldol condensation) of corresponding ringopening products (Nakagawa and Tomishige, 2012). Meanwhile, we did not observe any hydroxylmethylated product with a decreased acid loading of $1 \mu \mathrm{L}\left(0.01 \mathrm{mmol} \mathrm{H}^{+}\right)$and a reaction time of $4 \mathrm{~h}$ at $60^{\circ} \mathrm{C}$, further indicating that acid-catalyzed ring opening and condensation reactions was kinetically faster than the hydroxylmethylation reaction of EMF With an appropriate loading $\left(10 \mu \mathrm{L}, 0.1 \mathrm{mmol} \mathrm{H}^{+}\right)$of $\mathrm{HCl}$ aqueous solution, the effects of reaction temperature and time on the hydroxylmethylation efficiency of EMF were further investigated. Overall, increasing the reaction temperature and time facilitated the EMF conversion and the formation of hydroxylmethylated product (EMFM). When the reaction temperature was $30^{\circ} \mathrm{C}$, no desired product was detected in the early stage of the reaction $(<4 \mathrm{~h})$ (Figure 1B); when the reaction temperature was increased to $40^{\circ} \mathrm{C}$, the EMFM yield gradually increased with the increase of the reaction time (Figure 1C). At an elevated reaction temperature of $50^{\circ} \mathrm{C}$, the maximum yield $(28.3 \mathrm{~mol} \%)$ of EMFM was achieved at $5 \mathrm{~h}$ (Figure 1D) but the EMFM yield slightly decreased at an extended reaction time $(6-8 \mathrm{~h})$ likely due to the condensation of furanics. With the same loading $\left(10 \mu \mathrm{L}, 0.1 \mathrm{mmol} \mathrm{H}^{+}\right)$of $\mathrm{HCl}$ aqueous solution, severe condensation or carbonization was observed when a higher temperature of $60^{\circ} \mathrm{C}$ was used. Highmolecular-weight products $(300-1,000 \mathrm{Da})$ (Entry 1 in
Supplementary Table S1) observed in the GPC results (Supplementary Figure S9) confirmed the occurrence of condensation during the reaction. In the reaction system of $\mathrm{HCl}$ and formaldehyde aqueous solution, these results indicated that a high reaction temperature could significantly increase the rate of side reactions (furan ring opening and condensation), resulting in a serious decrease in the yield of the hydroxylmethylated product.

\section{Synthesis of BHMFM and BHMFD From FA}

Under the similar conditions, above results promoted us to conduct the hydroxymethylation reaction of FA (entry 1 in Supplementary Table S1) with formaldehyde and $\mathrm{HCl}$ aqueous solutions in 1,4-dioxane at $50{ }^{\circ} \mathrm{C}$ for 1 and $5 \mathrm{~h}$. However, no hydroxymethylated products were detected after replacing the EMF with FA (entry 11 in Supplementary Table S2). In addition to the furan ring opening (Liang et al., 2017) and aldol condensation of ring opening products, we infer that in the presence of water, acid could also catalyze the hydrolysis of the ester linkage of FA to form furfuryl alcohol, which could easily condense. Such side reactions could occur for the newly formed hydroxylmethylated products of FA. The hydroxyl groups could be protonated to form a carbonium ion, which could attack the electron-rich furan ring to form condensed products ( $\mathrm{He}$ et al., 2021).

To avoid the hydrolysis of the ester linkages of FA and condensation reactions caused by strong acid in the aqueous solution, we therefore explored the hydroxymethylation reaction of FA using a weak anhydrous acid (i.e., anhydrous acetic acid) and paraformaldehyde (Entry 1 in Supplementary Table S2) instead of formaldehyde and $\mathrm{HCl}$ aqueous solutions. In an anhydrous reaction medium, a hydroxymethylated product of FA (2,5-bis(hydroxymethyl)furan monoacetate (BHMFM)) was identified by MS (Supplementary Figure S4) and ${ }^{1} \mathrm{H}-\mathrm{NMR}$ (Supplementary Figure S5) under an elevated temperature from 80 to $120^{\circ} \mathrm{C}$. With acetic acid in the reaction system, BHMFM was further esterified by acetic acid to produce another derivative, 2,5-bis(hydroxymethyl)furan diacetate (BHMFD), which was also confirmed by MS (Supplementary Figure S6) and ${ }^{1} \mathrm{H}-\mathrm{NMR}$ (Supplementary Figure S7). The results indicated that anhydrous acidic environment was favorable to the hydroxymethylation of FA while the aqueous reaction system (e.g., formaldehyde and $\mathrm{HCl}$ aqueous solution) was indeed not favorable to the hydroxylmethylation of FA under acidic conditions. At a mild reaction temperature of $80^{\circ} \mathrm{C}$, no BHMFM was detected within $3 \mathrm{~h}$. After that, BHMFM yield gradually increased with the extension of the reaction time (Figure 2B). When the reaction temperature was elevated to $100{ }^{\circ} \mathrm{C}$, BHMFM was quickly formed within $2 \mathrm{~h}$. Further increasing the reaction time resulted in decreased BHMFM yields (Figure 2C). This was mainly caused by the esterification of BHMFM to form BHMFD (Figure 2A). The acetylation of BHMFM to form BHMFD was more evident at $120^{\circ} \mathrm{C}$ than those at $100^{\circ} \mathrm{C}$ (Figure 2D). At a reaction time of $3 \mathrm{~h}$ at $120^{\circ} \mathrm{C}, \mathrm{BHMFM}$ was almost completely esterified to form BHMFD while the total yield of BHMFM and BHMFD was not improved at elevated reaction temperatures $\left(>120{ }^{\circ} \mathrm{C}\right)$. 
A

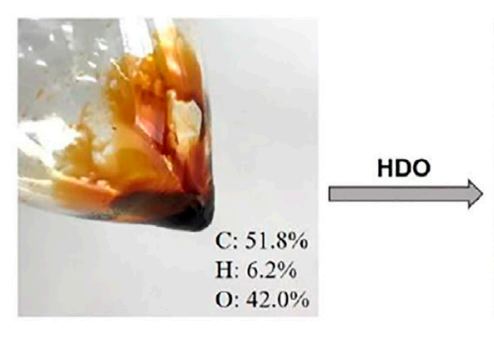

B
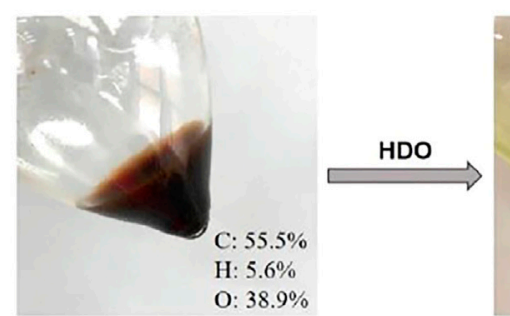
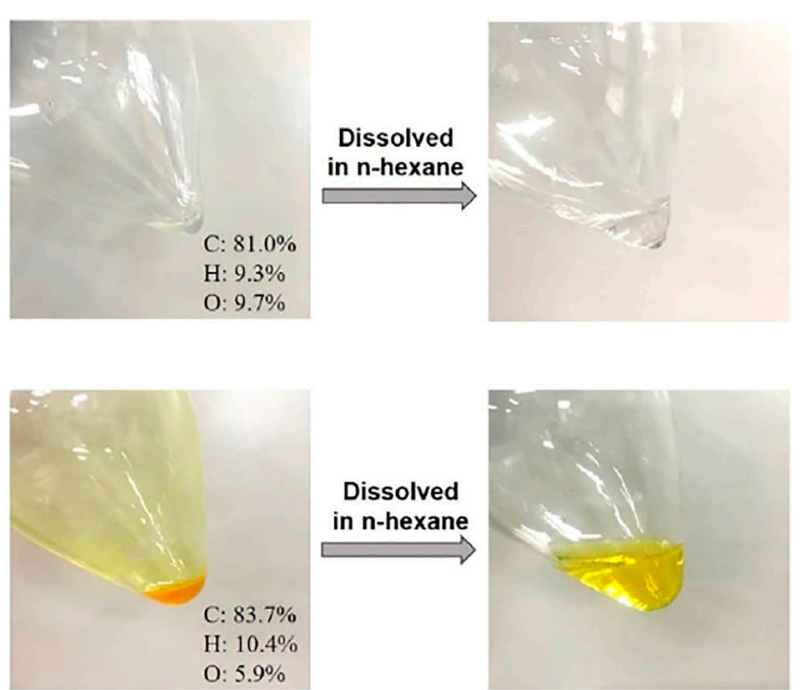

FIGURE 3 | Images of condensed furanic products before and after HDO reactions. (A) The condensed mixture resulting from FA reacting with paraformaldehyde in $\mathrm{CH}_{3} \mathrm{COOH}$; (B) The condensed mixture resulting from EMF reacting with formaldehyde in 1,4-dioxane.

In-situ esterification could be considered as a protection strategy to prevent the condensation of BHMFM in acidic reaction system. However, the yield of BHMFM or BHMFD was not beyond $30 \mathrm{~mol} \%$ under the investigated conditions. Similar to the hydroxymethylation reaction of EMF, this would be mainly caused by different reaction rates of target (hydroxymethylation and esterification) and side reactions (dehydration, furan ring opening and condensation). First, it is difficult to improve the yields of BHMFM and BHMFD if the ring-opening rate of furan rings is faster than the hydroxymethylation rate of FA. Second, even the reaction rate of hydroxymethylation is faster than that of furan ring opening, newly formed hydroxymethyl group could be immediately protonated by hydrogen proton and dehydrated to produce carbonium ions in an acidic medium. Carbonium ions are active components that would initiate methylene-bridging condensation products, making the esterification of BHMFM difficult and also resulting in low yields of BHMFM and BHMFD.

\section{Synthesis of Hydrocarbon Fuels From Condensed Furanic Products}

From the perspective of kinetics, it can be seen that no matter how to optimize the reaction conditions, part of the furanics would condense to form higher-molecular-weight products (Supplementary Figure S9). Such an issue is also associated with many other studies involving HMF, furfural and their derivatives (Randolph et al., 2018; Chang et al., 2020; Modugno and Titirici, 2021). In addition to the synthesis of hydroxylmethylated products, we tentatively hydrogenated the condensed furanic products into hydrocarbon fuels in order to improve carbon utilization. Oligomerization of furanics followed by hydrodeoxygenation (HDO) reactions to produce hydrocabron fuels and lubricants have been intensively studied (Li et al., 2021). Herein, we investigated the possibility of hydrogenating the condensed furanics into hydrocarbon fuels.

The residue after the removal of furanic monomers via hexane extraction was considered as condensed mixture and used to produce hydrocarbon fuels via hydrodeoxygenation. According to a previously reported procedure (Huber et al., 2005), we used two-step HDO reactions to remove oxygen-containing functional groups from the condensed furanics. The first step was to partially hydrogenate the oxygenated furanic products to increase its solubility in the hydrophobic solvents that was used in the second step; the second step was to near completely remove oxygen from the oxygenated furanic products to obtain hydrocarbon fuels. After these HDO reactions, the color of EMF- and FA-derived oily products changed from dark to colorless and light yellow (Figure 3), respectively. Moreover, the oily products produced by HDO reactions could be well dissolved in $n$-hexane to form homogeneous and transparent solutions (Figure 3), indicating that these condensed furanics were successfully hydrodeoxygenated and the resultant oily products can be possibly used as drop-in hydrocarbon fuels or additives in gasoline or diesel.

The element contents of the condensed furanics before and after HDO reactions were further analyzed to confirm the occurrence of hydrodeoxygenation. It can be seen from Figure 3 that, the oxygen content of EMF-derived oily products was significantly decreased from 38.9 to $5.9 \%$ after $\mathrm{HDO}$ reactions, at which the contents of $\mathrm{C}$ and $\mathrm{H}$ in $\mathrm{HDO}$ products were increased from initial 55.5 and 5.6\%-83.7 and 10.4\% (Supplementary Table S3), respectively. Similar results were also obtained for that of FA-derived oily products (Supplementary Table S3), where O content decreased from 
42.0 to $9.7 \%$ and $\mathrm{C}$ and $\mathrm{H}$ contents increased from 51.8 to $6.2 \%-81.0$ and $9.3 \%$, respectively. These results indicate that oxygen was efficiently removed from EMF- and FA-derived condensates to form hydrophobic hydrocarbon fuels with good solubility in n-hexane. As it was known, HMF-involved reactions generally lead to many condensed products, this preliminary study opened up a new way to produce hydrocarbon fuels from condensed furanics products which has been considered as waste, improving the carbon utilization efficiency.

\section{CONCLUSION}

In this study, HMF derivatives, i.e., EMFM, BHMFM and BHMFD were synthesized using FA and EMF as raw materials through acidcatalyzed hydroxymethylation, proving the possibility of making sixcarbon HMF derivatives from five-carbon furfural derivatives. Although such pathway is feasible, achieving high yields of hydroxymethylated products in these reactions seems challenging due to the paralleled ring-opening reaction of furanics and condensation of the resulting ring-opening products. Therefore, future research would be development of more efficient catalysts or reaction systems to suppress these side reactions to improve the selectivity of the targeted products. Alternatively, the by-products of these reactions were successfully converted into oil through hydrodeoxygenation reactions in order to improve the carbon utilization of furanics. This study provides a new route to synthesize the intermediate products of BHMF and FDCA from easily available raw materials and also prove the feasibility of converting condensed furanic by-products into drop-in hydrocarbon fuels.

\section{REFERENCES}

Chang, H., Bajaj, I., Huber, G. W., Maravelias, C. T., and Dumesic, J. A. (2020). Catalytic Strategy for Conversion of Fructose to Organic Dyes, Polymers, and Liquid Fuels. Green. Chem. 22 (16), 5285-5295. doi:10.1039/d0gc01576h

Chen, D., Cang, R., Zhang, Z.-D., Huang, H., Zhang, Z.-G., and Ji, X.-J. (2021). Efficient Reduction of 5-hydroxymethylfurfural to 2, 5-bis (Hydroxymethyl) Furan by a Fungal Whole-Cell Biocatalyst. Mol. Catal. 500 (3), 111341-111347. doi:10.1016/j.mcat.2020.111341

Cheng, F., Guo, D., Lai, J., Long, M., Zhao, W., Liu, X., et al. (2021). Efficient Basefree Oxidation of 5-hydroxymethylfurfural to 2,5-furandicarboxylic Acid over Copper-Doped Manganese Oxide Nanorods with Tert-Butanol as Solvent. Front. Chem. Sci. Eng. 15 (4), 960-968. doi:10.1007/s11705-020-1999-5

Elsayed, I., Jackson, M. A., and Hassan, E. B. (2020). Hydrogen-Free Catalytic Reduction of Biomass-Derived 5-Hydroxymethylfurfural into 2,5Bis(hydroxymethyl)furan Using Copper-Iron Oxides Bimetallic Nanocatalyst. ACS Sustain. Chem. Eng. 8 (4), 1774-1785. doi:10.1021/ acssuschemeng.9b05575

Gao, T., Gao, T., Fang, W., and Cao, Q. (2017). Base-free Aerobic Oxidation of 5hydroxymethylfurfural to 2,5-furandicarboxylic Acid in Water by HydrotalciteActivated Carbon Composite Supported Gold Catalyst. Mol. Catal. 439, 171-179. doi:10.1016/j.mcat.2017.06.034

Guan, W., Zhang, Y., Chen, Y., Wu, J., Cao, Y., Wei, Y., et al. (2021). Hierarchical Porous Bowl-like Nitrogen-Doped Carbon Supported Bimetallic AuPd Nanoparticles as Nanoreactors for High Efficient Catalytic Oxidation of HMF to FDCA. J. Catal. 396, 40-53. doi:10.1016/j.jcat.2021.02.012

Gupta, D., Kumar, R., and Pant, K. K. (2020). Hydrotalcite Supported Bimetallic (Ni-Cu) Catalyst: A Smart Choice for One-Pot Conversion of Biomass-Derived

\section{DATA AVAILABILITY STATEMENT}

The original contributions presented in the study are included in the article/Supplementary Material, further inquiries can be directed to the corresponding authors.

\section{AUTHOR CONTRIBUTIONS}

LS and CL developed the concept; XLV conducted the synthesis and GC analysis of the products; XC conducted the separation of products; JL conducted the elemental analysis; XLU conducted the NMR analysis and wrote the manuscript. LS and CL modified the concept and proofread the manuscript for language and technicalities.

\section{FUNDING}

This work was supported by National Natural Science Foundation of China (31870559, 31901262 and 32071716), Fujian Provincial Department of Science and Technology (2018J01590 and 2019J01387), Outstanding Youth Funding (xjq201923) of Fujian Agriculture and Forestry University.

\section{SUPPLEMENTARY MATERIAL}

The Supplementary Material for this article can be found online at: https://www.frontiersin.org/articles/10.3389/fbioe.2022.851668/ full\#supplementary-material

Platform Chemicals to Hydrogenated Biofuels. Fuel 277, 118111-111821. doi:10.1016/j.fuel.2020.118111

He, J., Qiang, Q., Liu, S., Song, K., Zhou, X., Guo, J., et al. (2021). Upgrading of BiomassDerived Furanic Compounds into High-Quality Fuels Involving Aldol Condensation Strategy. Fuel 306, 121765-121789. doi:10.1016/j.fuel.2021.121765

Hu, K., Zhang, M., Liu, B., Yang, Z., Li, R., and Yan, K. (2021). Efficient Electrochemical Oxidation of 5-hydroxymethylfurfural to 2,5furandicarboxylic Acid Using the Facilely Synthesized 3D Porous WO3/Ni Electrode. Mol. Catal. 504, 111459-111467. doi:10.1016/j. mcat.2021.111459

Huber, G. W., Chheda, J. N., Barrett, C. J., and Dumesic, J. A. (2005). Production of Liquid Alkanes by Aqueous-phase Processing of Biomass-Derived Carbohydrates. Science 308 (5727), 1446-1450. doi:10. 1126/science.1111166

Kandasamy, P., Gogoi, P., Venugopalan, A. T., and Raja, T. (2021). A Highly Efficient and Reusable Ru-NaY Catalyst for the Base Free Oxidation of 5Hydroxymethylfurfural to 2,5-Furandicarboxylic Acid. Catal. Today 375, 145-154. doi:10.1016/j.cattod.2020.05.009

Li, S., Ma, Q., Zhong, W., Zhao, X., Wei, X., Zhang, X., et al. (2021). One-pot Hydrodeoxygenation of Bioderived Furans into Octane at Low Temperatures via an Octanediol Route. Green. Chem. 23 (13), 4741-4752. doi:10.1039/ dlgc00916h

Liang, X., Haynes, B. S., and Montoya, A. (2017). Acid-Catalyzed Ring Opening of Furan in Aqueous Solution. Energy Fuels 32 (4), 4139-4148. doi:10.1021/acs. energyfuels.7b03239

McKenna, S. M., Leimkühler, S., Herter, S., Turner, N. J., and Carnell, A. J. (2015). Enzyme cascade Reactions: Synthesis of Furandicarboxylic Acid (FDCA) and Carboxylic Acids Using Oxidases in Tandem. Green. Chem. 17 (6), 3271-3275. doi:10.1039/c5gc00707k 
Modugno, P., and Titirici, M. M. (2021). Influence of Reaction Conditions on Hydrothermal Carbonization of Fructose. ChemSusChem 14 (23), 5271-5282. doi:10.1002/cssc.202101348

Nakagawa, Y., and Tomishige, K. (2012). Production of 1,5-pentanediol from Biomass via Furfural and Tetrahydrofurfuryl Alcohol. Catal. Today 195 (1), 136-143. doi:10.1016/j.cattod.2012.04.048

Randolph, C., Lahive, C. W., Sami, S., Havenith, R. W. A., Heeres, H. J., and Deuss, P. J. (2018). Biobased Chemicals: 1,2,4-Benzenetriol, Selective Deuteration and Dimerization to Bifunctional Aromatic Compounds. Org. Process. Res. Dev. 22 (12), 1663-1671. doi:10.1021/acs.oprd.8b00303

Rao, K. T. V., Hu, Y., Yuan, Z., Zhang, Y., and Xu, C. C. (2021). Green Synthesis of Heterogeneous Copper-Alumina Catalyst for Selective Hydrogenation of Pure and Biomass-Derived 5-hydroxymethylfurfural to 2,5-bis(hydroxymethyl)furan. Appl. Catal. A: Gen. 609, 117892-117936. doi:10.1016/j.apcata.2020.117892

Sajid, M., Zhao, X., and Liu, D. (2018). Production of 2,5-furandicarboxylic Acid (FDCA) from 5-hydroxymethylfurfural (HMF): Recent Progress Focusing on the Chemical-Catalytic Routes. Green. Chem. 20 (24), 5427-5453. doi:10.1039/ c8gc02680g

Shuai, L., Amiri, M. T., Questell-Santiago, Y. M., Héroguel, F., Li, Y., Kim, H., et al. (2016). Formaldehyde Stabilization Facilitates Lignin Monomer Production during Biomass Depolymerization. Science 354 (6310), 329-333. doi:10.1126/ science.aaf7810

Yang, Y., and Mu, T. (2021). Electrochemical Oxidation of Biomass Derived 5hydroxymethylfurfural (HMF): Pathway, Mechanism, Catalysts and Coupling Reactions. Green. Chem. 23 (12), 4228-4254. doi:10.1039/d1gc00914a

Ye, L., Han, Y., Wang, X., Lu, X., Qi, X., and Yu, H. (2021). Recent Progress in Furfural Production from Hemicellulose and its Derivatives: Conversion
Mechanism, Catalytic System, Solvent Selection. Mol. Catal. 515, 111899-111910. doi:10.1016/j.mcat.2021.111899

Yu, L., Chen, H., Wen, Z., Jin, M., Ma, X., Li, Y., et al. (2021). Efficient Aerobic Oxidation of 5-Hydroxymethylfurfural to 2, 5-Furandicarboxylic Acid over a Nanofiber Globule La- $\mathrm{MnO}_{2}$ Catalyst. Ind. Eng. Chem. Res. 60 (4), 1624-1632. doi:10.1021/acs.iecr.0c05561

Zhang, J., Gong, W., Yin, H., Wang, D., Zhang, Y., Zhang, H., et al. (2021). In Situ Growth of Ultrathin $\mathrm{Ni}(\mathrm{OH})_{2}$ Nanosheets as Catalyst for Electrocatalytic Oxidation Reactions. ChemSusChem 14 (14), 2935-2942. doi:10.1002/cssc.202100811

Conflict of Interest: The authors declare that the research was conducted in the absence of any commercial or financial relationships that could be construed as a potential conflict of interest.

Publisher's Note: All claims expressed in this article are solely those of the authors and do not necessarily represent those of their affiliated organizations, or those of the publisher, the editors and the reviewers. Any product that may be evaluated in this article, or claim that may be made by its manufacturer, is not guaranteed or endorsed by the publisher.

Copyright (C) 2022 Lv, Luo, Cheng, Liu, Li and Shuai. This is an open-access article distributed under the terms of the Creative Commons Attribution License (CC BY). The use, distribution or reproduction in other forums is permitted, provided the original author(s) and the copyright owner(s) are credited and that the original publication in this journal is cited, in accordance with accepted academic practice. No use, distribution or reproduction is permitted which does not comply with these terms. 